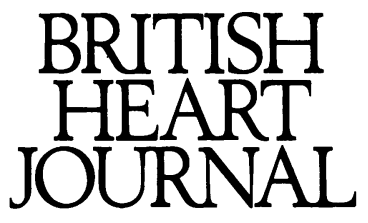

\title{
Editorial
}

\section{Gene therapy for cardiovascular disease}

The pace of recent developments in molecular biology has been unparalleled in modern medical history. Therapeutic manipulation of gene expression, considered to be in the realms of science fiction little more than a decade ago, is now being evaluated in clinical trials as a treatment for the genetic disorders that cause inborn errors of metabolism. The same technology is allowing investigators to consider the possibility of genetic manipulation, or gene therapy, as a treatment for acquired disorders, including atherosclerotic arterial disease, dilated cardiomyopathies, and restenosis after percutaneous vascular interventions. Two distinct approaches have been used as methods of influencing the molecular machinery of differentiated somatic cells. The first approach is gene therapy, which involves the introduction into cells of DNA encoding the production of a specific protein that has a local, regional, or systemic effect depending on whether it is retained within the cell or is actively secreted into the extracellular space. This approach may be used to replace proteins that are absent or defective owing to an inherited genetic abnormality or a spontaneous mutation, or to express therapeutic proteins with stimulatory or inhibitory activity as a treatment for acquired disorders.

The second molecular approach to cardiovascular therapeutics is the use of synthetic antisense oligonucleotides targeted to specific nuclear mRNA sequences. Oligonucleotides that are complementary, or antisense, to individual mRNA sequences bind to the sequence and prevent its translation. Inhibition of several cellular proto-oncogenes, including $c-m y b$, c-myc, nonmuscle myosin, and proliferating cell nuclear antigen (PCNA) has been shown to inhibit smooth muscle cell proliferation in vitro. ${ }^{1}$ Antisense oligonucleotides to c-myb have been successfully used to inhibit neointimal thickening in vivo in a balloon injured rat carotid artery model of restenosis. ${ }^{2}$ Although this approach has generated a great deal of interest and discussion, its clinical applicability remains limited by a relative lack of specificity, ${ }^{3}$ slow uptake across the cell membrane, and rapid intracellular degradation of the oligonucleotides. ${ }^{1}$ The use of antisense technology as a potential treatment for the prevention of restenosis has been reviewed elsewhere. ${ }^{1}$ The present editorial focuses on somatic gene therapy.

\section{Somatic gene therapy}

Various methods have been examined as means of introducing DNA into somatic cells. These include the use of viral vectors (in particular, retroviruses and adenoviruses), liposomes, direct injection, and physical methods such as electroporation. Viral vectors allow the uptake of foreign DNA through their interaction with specific membrane receptors. Replication-defective adenoviruses seem to be most efficient in transfecting mammalian cells but are associated with the shortest duration of expression of the foreign gene. In contrast to retrovirus-mediated DNA transfer in which the foreign DNA is randomly inserted into the host cell genome, DNA introduced by adenoviral vectors remains episomal, and is not incorporated into the host cell nucleus. This reduces the potential for insertional mutagenesis and malignant transformation, but reduces the duration of gene expression. In addition, since prior adenoviral infection is almost universal in the adult population, the use of these vectors carries the potential risk of inducing an immunological response to the introduced gene and its vector. Retrovirus-mediated gene transfer, on the other hand, is associated with minimal risk of immunogenicity and a long duration of expression, but it also has the theoretical potential for mutagenesis and a relatively low efficiency of transfection of non-proliferating cells. Liposomal gene transfer, in which the foreign DNA is coated with a cationic layer of lipid that binds to the target cell membrane, is a safe alternative with a modest efficiency and intermediate duration of gene expression.

\section{Clinical applications of gene transfer}

Although originally conceived as a means of curing inherited genetic diseases by replacing mutated genes in germ cells, gene therapy will have its greatest clinical impact in the immediate future as a means of replacing deficient proteins in differentiated somatic cells. This is because of the profound ethical and philosophical implications of germ cell manipulations. In the United States the first federally approved clinical trial of somatic gene therapy for a genetic disorder began in September 1990 as a treatment for adenosine deaminase deficiency, which causes severe combined immunodeficiency (SCID). ${ }^{4}$ As of February 1993, there were 25 federally approved trials underway, with other clinical trials planned or initiated in Europe and China. Preliminary results from some of the studies have been very encouraging. One clinical trial initiated at the University of Michigan under the direction of Dr James Wilson has involved patients with homozygous familial hypercholesterolaemia $(\mathrm{FH})$, a condition associated with an absence or deficiency of the hepatic low density lipoprotein (LDL) receptor and characterised by severe hypercholesterolaemia and premature atherosclerosis. Patients enrolled in the study were treated by ex-vivo retrovirus-mediated transfer of the gene encoding the normal human LDL receptor to cultured hepatocytes 


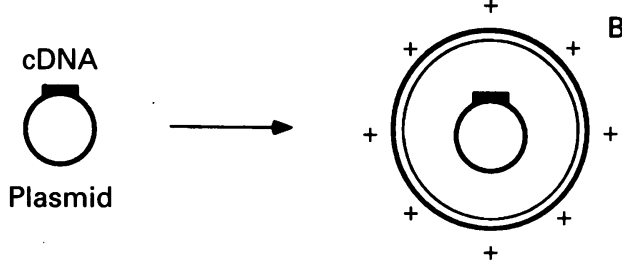

DNA-liposome complex

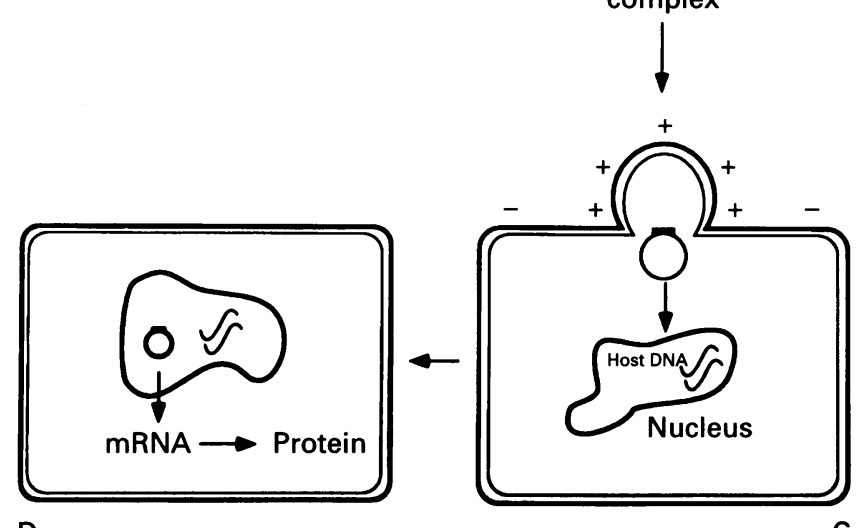

D

Figure 1 Liposome-mediated DNA transfer. The foreign DNA is initially introduced into the circular structure of an appropriate plasmid expression vector $(A)$. This vector is then mixed with cationic liposomes to form DNA-liposome complexes (B). This allows entry of the DNA through the cell membrane and its uptake by unknown mechanisms into the cell nucleus $(C)$. The foreign DNA is then processed in the same way as host DNA to produce the protein of interest (D).

that were isolated from a surgically resected segment of the patient's liver. ${ }^{5}$ The treated cells were then reintroduced into the liver by infusion into the portal vein through a catheter left in situ at the time of the hepatic resection. In the first patient treated, the procedure was associated with a rapid and sustained reduction in serum LDL, an increase in serum HDL, and a further improve-

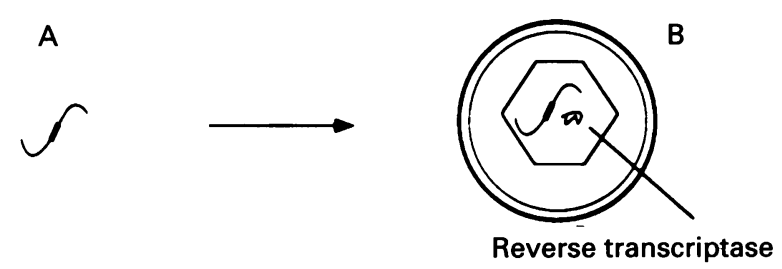

Provirus containing foreign gene

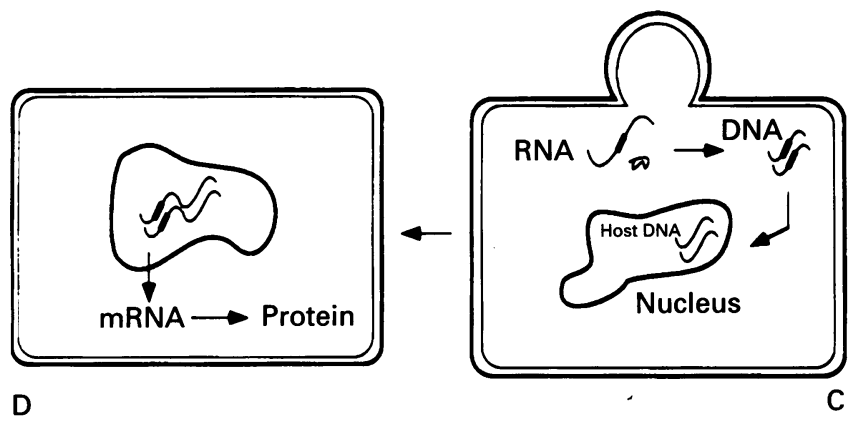

Figure 2 Retrovirus-mediated gene transfer. The foreign gene is initially substituted for a viral gene to form a provirus $(A)$ which is then packaged into the retroviral core $(B)$. The retrovirus binds to the target cell membrane via specific receptors and the viral RNA is introduced into the cytoplasm. The viral RNA and the foreign gene are then converted to $D N A$ by the viral enzyme reverse transcriptase $(C)$. The viral DNA and foreign gene are then inserted randomly into the host cell chromosome (D). This allows protein synthesis to occur through normal mechanisms. ment in lipid profile after treatment with lovastatin. ${ }^{5}$ Cells expressing the transgene were detected by in situ hybridisation of tissue obtained by liver biopsy four months after treatment. Clearly, if this approach is shown to be feasible and effective in larger studies it may be a major advance in the treatment of not only the few patients with homozygous $\mathrm{FH}$ and devastatingly accelerated atherosclerosis but potentially also of the many patients with the heterozygous form of the disease in whom a moderate degree of hypercholesterolaemia is also associated with premature atherosclerotic coronary and peripheral vascular disease.

The second potential role for gene therapy as a treatment for cardiovascular disease is as a means of inhibiting the development or progression of acquired disease processes, such as atherosclerosis, arterial thrombosis, or restenosis after percutaneous interventions. Because the acquired diseases are multifactorial in aetiology, this is without doubt a far greater challenge than replacing or augmenting deficient proteins associated with the inherited single gene defects. Fortunately, gene therapy also offers the opportunity to define better the role of key factors involved in these processes. In a series of studies performed in the laboratory of Dr Elizabeth Nabel, genes encoding individual growth factors, such as platelet derived growth factor (PDGF), ${ }^{6}$ fibroblast growth factor (FGF), ${ }^{7}$ and transforming growth factor (TGF) $\beta,{ }^{8}$ have been transferred to the intact vascular endothelium. Local expression of each mitogen was associated with the development of a neointima with histological features similar to those seen after vascular injury with distinct differences between the appearances of the neointima for each mitogen. Expression of TGF $\beta$ was associated with a greater degree of collagen matrix production than PDGF-induced neointimal thickening, ${ }^{8}$ and FGF expression was associated with the development of a prominent network of intimal capillaries ${ }^{7}$ consistent with prior observations that FGF induces angiogenesis. It is hoped that these and other studies will greatly improve our understanding of the complex series of events that follow vascular injury and lead to atherogenesis or restenosis. Only then can we begin to direct inhibitory genes at specific targets that might impede the progress of these disease processes.

In addition to attempts to treat cardiovascular diseases through the secretion of inhibitory factors, gene therapy also offers the potential for treating established acquired diseases by the local expression of stimulatory proteins. In a recent study, Takeshita and colleagues reported the induction of functionally significant collateral vessels by transfer of the gene encoding vascular endothelial growth factor (VEGF) in the rabbit hind limb rendered ischaemic by ligation of the external iliac and femoral arteries. ${ }^{9}$ Expression of this potent angiogenic factor by cells of the internal iliac arterial wall was associated with the development of an angiographically recognisable collateral network and an objective increase in perfusion of the ischaemic limb. This approach clearly holds great promise for the treatment of severe peripheral vascular disease and end stage ischaemic cardiomyopathies.

Challenges for clinical applicability of gene therapy The preclinical and early clinical experience described above suggest that the enthusiasm with which genetic manipulations have been accepted as potential treatments for both inherited and acquired diseases is undoubtedly warranted. It is important to recognise, however, that there are many major hurdles that must be overcome if this technology is to become routinely used 
in clinical practice. These considerations range from relatively minor technical concerns to far more difficult issues. Of greatest immediate concern for investigators treating patients with inherited single gene defects, in whom lifelong gene expression is desirable, are the questions of the efficiency of gene transfer and expression, the duration of gene expression, and the potential for mutagenesis and immunogenicity with repeated administration of the foreign gene and its vector. In the longer term the desire to obviate the need for tissue resection and ex vivo gene transfer will lead to the evaluation of intravenous gene delivery and the requirement for cell targeting and specificity of gene uptake and expression, for example by receptor-mediated DNA transfer ${ }^{10}$ and through the use of cell-specific promoters.

Investigators interested in the treatment of acquired diseases have the added and substantial challenge of identifying therapeutic genes that are effective not only in the controlled environment of in vitro systems but also in diseased tissues in vivo. Secondly, while ex vivo gene transfer may continue to be feasible for certain conditions such as the prevention of arterial vein graft disease, ${ }^{11}$ direct gene transfer to specific sites in the arterial tree will require modification of previously used techniques. Delivery systems used in peripheral arteries ${ }^{6-8}$ may not be useful for coronary arterial gene transfer because of the need to avoid myocardial ischaemia during balloon inflation and the inability to control runoff into the large coronary side branches. Studies addressing these issues through the use of hydrogel-coated or microporous balloon catheters, implantable intravascular devices, and retrograde delivery from the coronary sinus are currently underway. More difficult issues that must also be addressed include the need for in vivo regulation of the transgene, ${ }^{12}$ how to overcome constraints on the size of the gene that can be delivered, and how to rationalise the risk and cost benefits of this technically demanding treatment. Despite these limitations, however, there is every reason to be optimistic that gene therapy will not only be a first line treatment for certain inherited diseases, but may also become an invaluable adjunct or alternative to conventional mechanical and pharmacological treatments for acquired cardiovascular diseases.

Department of Internal Medicine,

University of Michigan Medical Center

Ann Arbor,

Michigan 48109, USA

1 Epstein SE, Speir E, Finkel T. Do antisense approaches to the problem of restenosis make sense? Circulation 1993;88:1351-3.

2 Simons M, Edelman ER, Dekeyser J-L, Langer R, Rosenberg RD. Antisense c-myb oligonucleotides inhibit intimal arterial smooth muscle Antisense c-myb oligonucleotides inhibit intimal arter
cell accumulation in vivo. Nature 1992;359:67-70.

3 Woolf TM, Melton DA, Jennings CGB. Specificity of antisense oligoWoolf TM, Melton DA, Jennings CGB. Specificity of antisen
nucleotides in vivo. Proc Natl Acad Science 1992;89:7305-9.

nucleotides in vivo. Proc Natl Acad Science 1992;89:7305-9.
Anderson WF. Human gene therapy. Science 1992;256:808-13.

4 Anderson WF. Human gene therapy. Science 1992;256:808-13. Grossman M, Raper SE, Kozarsky K, Stein EA, Engelhardt JF, Muller
DWM, et al. Liver-directed gene therapy in a patient with familial DWM, et al. Liver-directed gene therapy in a patien
hypercholesterolemia. Nature Genetics 1994;6:335-41.

6 Nabel EG, Yang Z, Liptay S, San H, Gordon D, Haudenschild CC, Nabel GJ. Recombinant platelet-derived growth factor B gene expression in porcine arteries induces intimal hyperplasia in vivo. $\mathcal{F}$ Clin Invest 1993;91:1822-9.

7 Nabel EG, Yang Z, Plautz G, Forough R, Zhan X, Haudenschild CC, et al. Recombinant fibroblast growth factor-1 promotes intimal hyperplasia and angiogenesis in vivo. Nature 1993;362:844-6.

8 Nabel EG, Shum L, Pompili VJ, Yang Z-Y, San H, Shu HB, et al. Direct transfer of transforming growth factor $\beta_{1}$ gene into arteries stimulates fibrocellular hyperplasia. Proc Natl Acad Sci USA 1993;90:10759-63.

9 Takeshita S, Zheng LP, Asahara T, Reissen R, Brogi E, Ferrara N, et al. In vivo evidence of enhanced angiogenesis following direct arterial gene In vivo evidence of enhanced angiogenesis following direct arterial gene [abstr]. Circulation 1993;88:I-476.

10 Wu GY, Wilson JM, Shalaby F, Grossman M, Shafritz DA, Wu CH. Receptor-mediated gene delivery in vivo: partial correction of genetic analbuminemia in Nagase rats. $\mathcal{F}$ Biol Chem 1991;266:14338-42.
11 Chen S-J, Wilson JM, Muller DWM. Adenovirus-mediated gene transfer of soluble vascular cell adhesion molecule to porcine interposition vein grafts. Circulation 1994;89:1922-8.

12 Kitsis RN, Buttrick PM, McNally EM, Kaplan ML, Leinwand LA. Hormonal modulation of a gene injected into rat heart in vivo. Proc Natl Acad Sci USA 1991;88:4138-42.

\section{Glossary}

Antisense DNA oligonucleotides-Synthesised DNA oligonucleotides that bind to complementary sequences of messenger RNA thereby inhibiting the translational synthesis of a targeted gene product (protein).

Complementary DNA ( $c D N A)$ - The DNA that has been copied from mRNA in a reaction catalysed by the enzyme reverse transcriptase.

Episomes-Genetic elements that can exist in stable form as pieces of nuclear DNA separate from the chromosomes.

Eukaryotic-Cells or organisms that contain a nucleus and that divide by mitosis (for example, yeast and mammalian cells).

Expression-Production of either a specific RNA or a peptide, depending on the context in which it is discussed.

Expression vector-A vector containing an inserted gene that is capable of inducing the synthesis of a large amount of protein coded by the gene when introduced into an appropriate host cell.

Gene-a sequence of DNA that carries the information encoding a polypeptide. It is the biological unit of heredity and is located at a defined locus on a particular chromosome.

Genome-The complete set of hereditary information in a species. It is composed of all the genes contained in the chromosomes.

Germ cells-Sperm and egg cells or their precursors. Foreign genes introduced into germ cells are passed on to the cells of the treated patient's progeny.

Hybridisation-The process of annealing two single strands of complementary nucleic acid to each other in order to form a double strand. Usually, one strand is a target sequence and the other is a labelled probe sequence.

In situ hybridisation-A procedure in which nucleic acid (DNA or RNA) of cells or tissue sections is denatured in situ so that reaction is possible with an added DNA or RNA probe. The added probe is labelled either radioactively or by an enzyme or protein marker and its hybridisation is detected by autoradiography, histochemistry, or immunohistochemistry.

Liposome-A synthetic vesicle consisting of a membrane of phospholipids used to facilitate transfection of DNA (or other macromolecules) into cells. The foreign DNA is usually incorporated into an appropriate plasmid expression vector.

Messenger (m)RNA-The RNA that carries genetic information copied from DNA to the cytoplasm to direct protein synthesis by specifying the sequence of amino acids.

Mutagenesis-The process by which the sequence of genomic DNA is changed, potentially leading to conversion of eukaryotic cells to a state of unrestrained growth.

Oligonucleotide-A polymer consisting of a small number of nucleotides.

Oncogene-Viral genetic material that, when introduced into a normal cell, can transform the host cell into a tumour cell. Each oncogene has a natural counterpart (proto-oncogene or cellular oncogene) in the normal eukaryotic genome with a similar DNA sequence.

Plasmid-A small, circular DNA molecule (1000-30000 base pairs) that replicates in bacteria and generally encodes proteins required for resistance to antibiotics or other toxins. Some plasmids are used as vectors, providing an essential tool of recombinant DNA technology. 
Promoter-The region of a gene that binds RNA polymerase and initiates and controls gene transcription.

Proto-oncogene-A gene present in the normal vertebrate cell genome. Many proto-oncogenes code for growth factors, growth factor receptors, or intracellular mediators of cell division.

Recombinant DNA-A DNA molecule produced by insertion of foreign DNA into an autonomously replicating DNA molecule such as a virus or plasmid.

Retrovirus-An RNA virus that propagates via conversion into DNA using the enzyme reverse transcriptase.

Reverse transcriptase-An enzyme found in retroviruses that can catalyse the synthesis of cDNA from mRNA.
Somatic cells-Cells other than sperm cells and egg cells. Gene transfer to these cells does not affect the offspring of the individual treated.

Transcription-The process of synthesising RNA on a DNA template.

Transfection-The transfer of new genetic material to a cell by the introduction of DNA.

Translation-The process of synthesising protein on an RNA template.

Vector-A molecule capable of autonomous replication, usually a bacteriophage or plasmid, that can grow in a particular host. It is used to receive, replicate, and allow the selection of the genomic DNA or cDNA of interest after introduction into the host.

\section{An unusual cause of poor cardiac output}

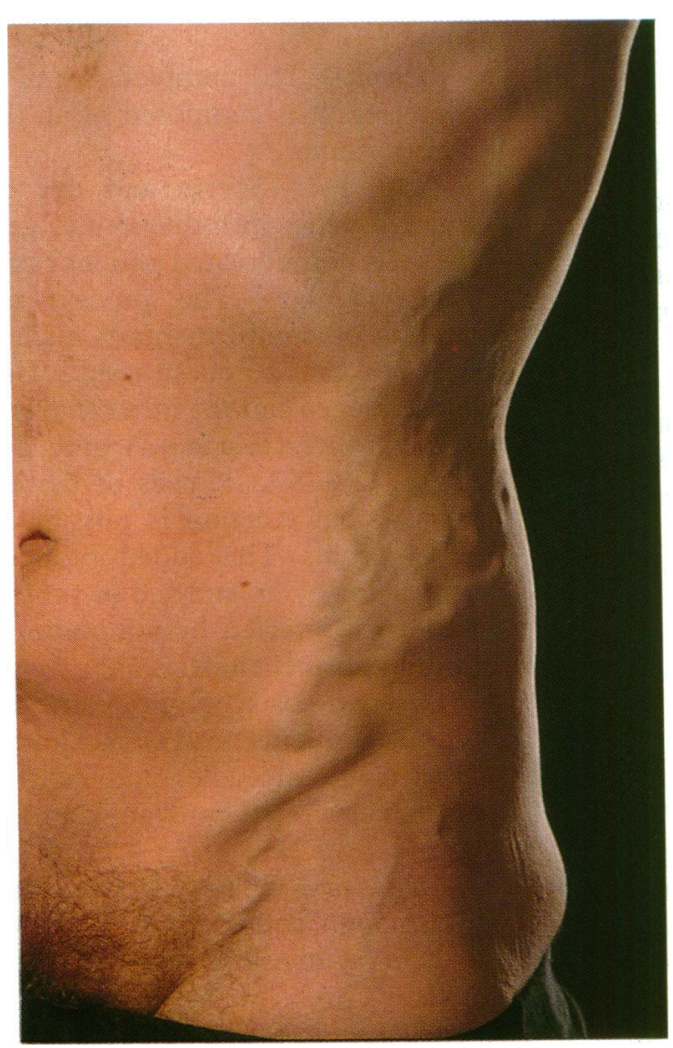

The photograph shows the development of a marked superficial collateral circulation in a patient in his late twenties who had had a retroperitoneal lymphoma with ligation of the inferior vena cava. I was asked to see him several years later because heart failure was suspected. His dyspnoea on exertion was caused by poor cardiac output which was the result of inadequate venous return. Left ventricular function was normal and his symptoms improved considerably when the dose of diuretic was reduced. Diuretics had been prescribed for peripheral leg oedema.

M K DAVIES 